\title{
Towards a Model for Discourse Ethics-based Digital Business Strategy Development
}

\section{Oliver Degner}

University of Duisburg-Essen

Essen, Germany

Email: oliver_degner@gmx.de

\section{Andreas Drechsler}

School of Information Management

Victoria University of Wellington

Wellington, New Zealand

Email: andreas.drechsler@vuw.ac.nz

\section{Abstract}

We develop a decision-making process model that implements generic discourse ethics and business ethics proposals specifically for ethical digital business strategy (DBS) development. The model's objective is to answer the question how DBS initiatives - with the potential to affect the wider society can be developed and justified considering economic, ecological and social criteria, based on consensus among the relevant stakeholders. We evaluate the model by examining two past DBS initiatives that were reported in the media as being controversial because of a lack of stakeholder consensus. With the model, we contribute a pragmatization of the business and discourse ethics literature for the IS discipline that covers the time before actual IS design and development decisions take place. Business and IT executives in practice can draw on the model to guide their DBS processes along ethical lines.

Keywords discourse ethics, business ethics, digital business strategy, digital strategy, strategy development 


\section{Introduction}

Digital transformation is a fashionable catchword used to describe several emerging and ongoing initiatives and trends where technological innovations drive fundamental changes to organizations, industries and societies - for instance, "big data" or the "internet of things". In this context, the development and subsequent implementation of a digital business strategy (DBS) (Bharadwaj et al. 2013) is an important instrument for an organization to forge new or convert and combine existing technological possibilities to create novel information systems that, upon wide-ranging user adoption, can transform the organization's products or processes.

Following Bharadwaj et al. (2013), we understand a DBS as an organizational strategy that - in a singleorganization perspective - strives to leverage digital resources in order to drive competitive advantage and strategic differentiation. Note that the DBS perspective is purposefully not limited to a traditional IS/IT strategy or IT organization perspective. Viewed through a wider lens, a DBS's successful execution can trigger further transformational effects across multiple organizations, an entire industry, local or even global society. However, such digital transformations of businesses, industries and society can (and most probably will) lead to both positive and negative effects for the various affected stakeholder groups. As such assessments of positive or negative effects are not absolute but a matter of values, there are ethical and moral considerations. And since some effects may profoundly affect people's working or personal lives, business ethics posits that the respective decision-makers consider these effects alongside their regular business-focused decision-making (Ulrich 2008). In the DBS context, such considerations would need to take place during the DBS development phase.

However, Mingers and Walsham (2010) have noted that ethics is underrepresented in the IS discourse. To improve matters, they advocate pragmatization as a first step, i.e. applying the sometimes abstract and idealized deliberations in the ethics discourse to concrete situations (for instance, by embedding them in IS methodologies and approaches). In particular, these authors highlight the potential of Habermasian (1990) discourse ethics to achieve informed consensus among affected stakeholders in order to ethically legitimize IS decisions. However, much work on ethics in the IS discipline so far has focused on IS development or design decisions (e.g., Myers and Venable 2014). In the digital transformation context, we acknowledge that the consideration of ethical issues during IS design plays an important role. However, we also posit that the IS design phase may be "one step too late" to start incorporating ethics into decision-making. We surmise that, during the design phase of actual systems, it can be difficult to account for aspects that should have been considered during the development of the overarching strategy or policy that led to the authorization of these systems' design initiatives. Simultaneously, since digital transformation and DBS formulation are highly informed and driven by technical opportunities and potentials, we consider ethical considerations during the DBS formulation stage a matter for the IS discipline to tackle. To our knowledge, there has not yet been such an initiative.

Against this backdrop, we take a first step towards the pragmatization of the ethics discourse for DBS development. In particular, we contribute a discourse ethics-based process model that integrates an engaged discourse with stakeholders on ethical matters into the DBS development process. We illustrate how the model can be used in an ex-post perspective to reconstruct and analyse past digital business strategy decisions and executions that did not sufficiently address ethical implications and subsequently led to negative effects on the company and the customers. We also posit that IT and business executives can draw on our model to incorporate ethical considerations during their future DBS development and execution. The model can further serve to highlight inherent tensions between DBS characteristics and ethical considerations. We develop the model following a review of selected ethics literature and evaluate it by reconstructing past digital business strategy executions that received media coverage because ethical implications were not adequately addressed.

The remainder of the paper is structured as follows: In the second section, we present our theoretical foundations, namely digital business strategies and discourse and integrative business ethics. In the third section, we discuss the resulting ethical implications for digital business strategy decision-making and develop our process model. In the fourth section, we evaluate the model and discuss our contributions in the fifth section, along with a conclusion.

\section{Theoretical Foundations}

\subsection{Digital business strategy}

With the increasing role of technology in organizations and the rising potential for new technological developments to transform businesses and entire domains, the strategic perspective in recent years has 
moved beyond traditional IS/IT strategic perspectives towards a consideration of a comprehensive digital business strategy (Bharadwaj et al. 2013). In particular, these authors identify four themes to take into account for DBS formulation: 1) the DBS scope, 2) the DBS scale, 3) the DBS development and execution speed and 4) the sources of value-creation and capture. The DBS scope addresses the extent to which traditional boundaries (between business and IT, different business functions or different organizations along the supply chain) are crossed. The DBS scale addresses the possibility of the rapid upscaling of different facets of digital product or service provision. The DBS development and execution speed comprises issues such as decision-making or product launch speed, since digital first-movers tend to be able to exert substantial and lasting influence on a particular market or industry. Lastly, the sources of value-creation and capture address new perspectives on business model development, where, for instance, one layer (such as applications) may be provided for free in order to monetize higher-order layers (such as services or advertising).

However, Bharadwaj et al. (2013) do not cover the actual DBS development and implementation processes in greater detail. Complementary articles tend to focus on single organizations' individual economic competitiveness within industries (Grover and Kohli 2013). However, implicitly or explicitly, DBSes also have the potential to affect and transform entire industry sectors or parts of society, including personal lives, on smaller or larger scales. These transformations can be triggered by the provision of technology itself (for instance, smartphones and social media / communication apps that lead to some people paying greater attention in their lives to their devices than to the real world) or by embedding technology in existing services (for instance, when health insurance companies give discounts to customers who live healthy lifestyles, as evidenced by apps automatically tracking and reporting health-related personal data to the insurance company). We posit that these ripple effects, as we will subsequently call them, of a DBS have the clear potential to be of a different quality and magnitude than ripple effects of traditional business strategy formulation and execution. For this reason, we think that the DBS development warrants special attention from an ethics perspective.

\subsection{Discourse ethics and integrative business ethics}

Our model has its roots in Peter Ulrich's (2008) proposal for integrative business ethics. The cornerstone of his proposal is that economic and ethical aspects of decision-making are not to be seen as taking place in separate worlds or value systems and that business ethics does not merely act as an ex-post corrective to business decisions. Instead, economic interests and ethical aspects are to be considered 'as equals' during organizational decision-making. In practical terms, this goal is achievable through applied discourse ethics. Discourse ethics goes back to Habermas (1990) and Apel (1980) and, in a nutshell, proposes to strive for an ideal communication setting in which all participants engage in a discourse on the moral and ethical implications of planned actions. Note that Ulrich (2008) develops his integrative business ethics approach through an analysis and synthesis of a great number of other researchers' works. However, this paper's page limit prevents us from having a finer-grained consideration and discussion of the respective sources.

The first step during this discourse on moral and ethical implications is to understand each other's interests and values. On this foundation, actors can inform and legitimize subsequent actions through achieved consensus. In other words, discourse ethics is not about normative but procedural ethics. In our context, the actors comprise any organization seeking to formulate and implement a DBS. Following the idea of integrative business ethics, stakeholders affected by the DBS are to be involved in a comprehensive discourse about the DBS before the organization takes the final decision in consensus with the affected stakeholders. Stakeholders include all groups and individuals in society that are or may be affected in any way, directly or indirectly, by the DBS implementation. Engaging the stakeholders in an open discourse also implies that the ethical considerations do not serve the main purpose of reducing the transactional or implementation costs of rational business decisions. Instead, the stakeholders' goals and values, together with the organization's own goals and values, are considered ex-ante in a consensus-achieving process and thus they all have an impact on the particular business decision's outcome. A reduction in transactional implementation costs may be a side-effect, however.

We see integrative business ethics as especially suited to and appropriate for DBS development, since subsequent DBS implementations may well quickly exert a 'normative power of the real', i.e. the successful implementation of DBS can lead to new technologies that rapidly disrupt extant social patterns so that they become a de facto standard for members of organizations and society to use, as the examples of social networks and instant messengers illustrate. It may well be the case that the respective members of organizations or society automatically have to accept any ethically questionable side-effects (such as having no real choice in providing personal data or being unable to communicate anonymously or over encrypted channels) or, alternatively, are negatively affected in their lives (such as by being 
excluded from regular social exchanges that have moved from face-to-face conversations or phone calls to online messages). These examples illustrate that ex-post corrections on ethical grounds of successful disruptive DBSes are almost impossible to achieve and thus further support the notion that the most appropriate place for ethical considerations is indeed the DBS development phase.

Pursuing this kind of integrative business ethics ultimately leads to an ethical legitimization and justification of organizational decisions such as the decision to pursue a particular DBS. Such a legitimization corresponds to one of Mingers and Walsham's (2010) proposed pathways for applying ethics to the IS field. On the economic side, the resulting benefits compared with traditional DBS development and implementation can include higher market acceptance, a subsequently even larger transformative disruption due to this higher acceptance and, consequently, a more sustained competitive advantage compared with competitors whose values are less shared or aligned with customers and users. On the ethical side, the benefits include legitimized and justified DBS decisions, as well as greater transparency about the implications and consequences of the directions that technology and business-driven organizational and societal development and transformations take.

\section{A Model for Discourse Ethics-based Digital Business Strategy Development}

While Ulrich (2008) considers specific organizational prerequisites that need to be in place to foster an effective integration of business ethics in organizational decision-making (e.g., establishing an ethical culture, aligning management systems to the ethical culture, formally empowering employees to participate in the discourse or establishing institutional safeguards), he does not discuss an immediately actionable process to conduct consensus-oriented organizational decision-making. We therefore adapt Ulrich's (2008) general recommendations to the DBS formulation setting and propose a concrete DBS development model that incorporates these recommendations on a more actionable level (cf. Figure 1).

First, we posit that any concrete, formulated DBS is an expression of a particular vision of how digitalization can transform the organization, its products or markets (step 1). Following Ulrich's (2008) proposal, an initial formulated DBS (step 2) needs to be subjected to an open discourse (step 3) within the organization and among affected stakeholders before it is implemented. The stakeholders can originate in civil society but also in the state and the business sectors (Marrewijk 2003). The civil society stakeholders include those individuals directly affected by a technology, as well as groups that act as representatives, such as NGOs. State representatives can be interested in maintaining desired or prevailing values within a state or, more concretely, need to be involved regarding existing laws that do not adequately account for new technologies, or where no regulations exist yet (take, for instance, the use of camera drones and its limitations). Also, business partner organizations may be affected by intended transformative changes to the supply chain. Moreover, industry associations may have interests regarding matters of self-governance/self-regulation or in maintaining industry values.

After the selection, the stakeholders assess the DBS regarding their implications for themselves (step 4). Stakeholders are influenced by their cultural (national culture, but also generational culture, especially in the digital context) and overarching ethical norms. The literature proposes three criteria to consider for such implication assessments: economical, ecological and societal values (Marrewijk 2003). While discourse ethics highlights the importance of an open dialogue, we propose complementing the dialogue with an initial rating of DBS implications for each stakeholder group based on these three criteria in order to make any divergent perceptions and assessment transparent and comparable. For this triangle diagram, we propose a seven-point scale that accounts for slightly, moderately or significantly positive or negative implications with a neutral point in the middle. The subsequent discourse (step 5) serves the purpose of highlighting reasons for the given ratings. In turn, the ratings can be changed based on a modified DBS proposal or enhanced understanding of DBS implementation implications.

In case no stakeholder group sees any negative implications for them in any of the three criteria, or if the DBS can be modified (step 6) so that the negative implications can be avoided (while the DBS still retains positive implications for the proposing organization, including the implementation of the overarching vision), the legitimization goal is achieved, and the organization can proceed with DBS implementation (step 7a). If the DBS cannot be modified to account for the identified negative implications for a stakeholder group, the organization can achieve a legitimization of their decision not to pursue the DBS (step 7b). However, there can also be a lack of consensus after the discourse. While this is, from an ethics standpoint, the least desirable outcome, the organization is, of course, free to implement the DBS anyway (step 7c), but this time without legitimization. Nevertheless, the discourse may still produce concrete open questions (steps $8 \mathrm{~b}-8 \mathrm{~d}$ ) for each of the stakeholder groups to further 
debate and likewise questions for the transcendental ethics discourse beyond individual stakeholder interests (step 8a). The corresponding answers can then influence and inform further discourses.

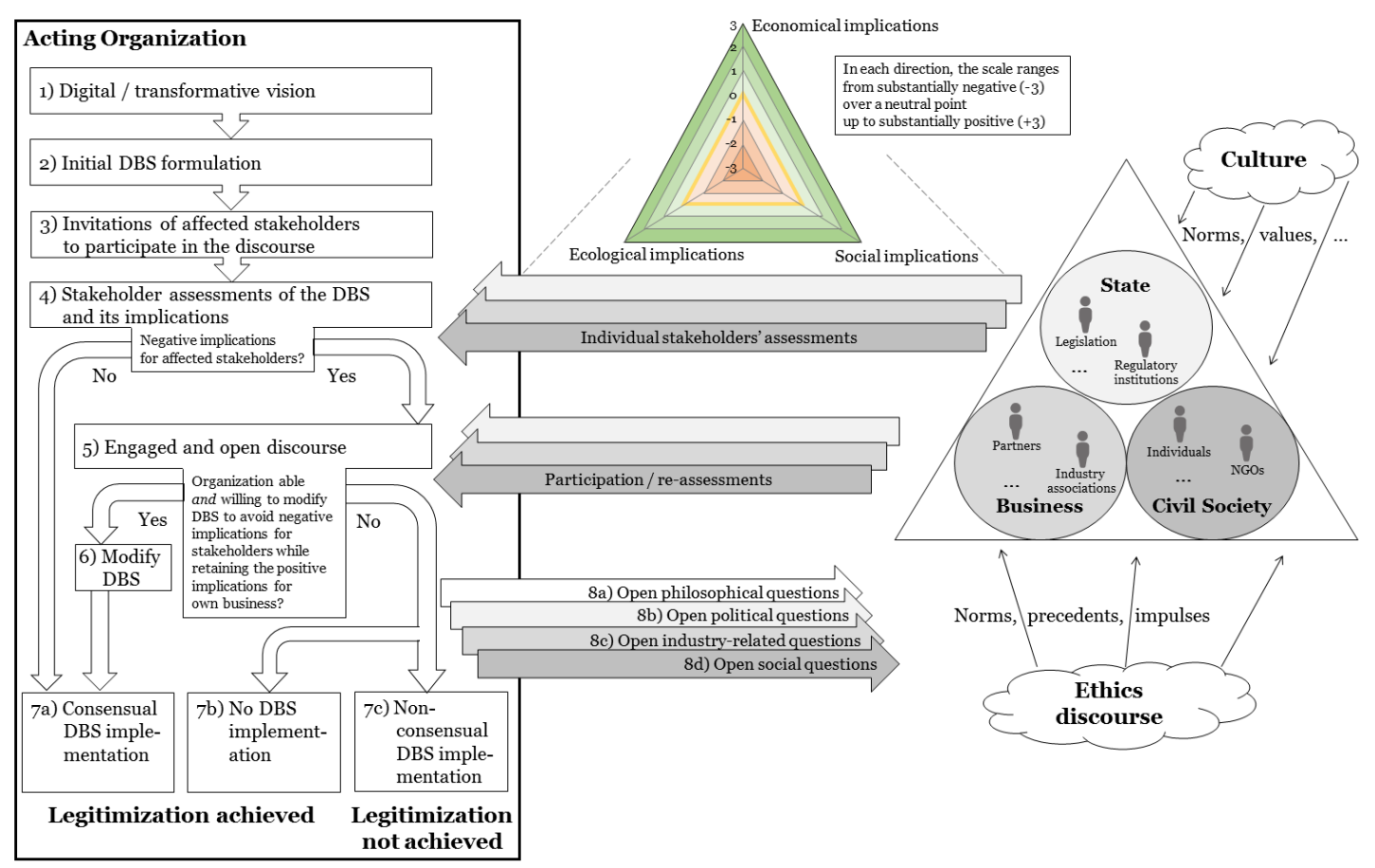

Figure 1. Process model for integrating business ethics considerations to DBS development

\section{Model Evaluation}

For the purpose of evaluating the model shown in Figure 1, we draw on media reports on two cases where two fashion labels (Peuterey and Gerry Weber) attached RFID tags to their clothing (Beuth 2012). The economic benefits include facilitating logistics during the supply chain, stocktaking within the outlet stores and preventing theft (Grone 2012). However, as a ripple effect, unremoved RFID tags within clothes also allow the identification of individuals and the tracking of their movements based on the tag's unique ID, which can be seen as endangering personal privacy (Digitalcourage e.V. 2011). We consider the two cases of RFID use as two instances of an overarching DBS that sought to digitalize complex logistics chains in order to increase efficiency and reduce transaction cost (steps 1 and 2). On a grander scale among other transformative DBSes, we see these two attempts as instances where the privacy of stakeholders' personal data (here, customer location and movements) is negatively affected by economically driven digitalization attempts. Note that our evaluation is solely ex-post (we had not yet formulated our model when these events occurred) and relies on our interpretation of media reports.

In the first case, the fashion label Peuterey decided to attach RFID tags without any discourse, legitimization or even announcement. It even added a note to the tag warning the customer against removing the label. This approach resulted in public damage to Peuterey's image, including when it received a well-publicized negative award for privacy violations handed out by the German NGO FoeBuD e.V. (now Digitalcourage e.V. (2011)). We can only speculate about concrete positive or negative economic effects for Peuterey.

In the second case, the fashion label Gerry Weber also decided to attach RFID tags to its clothing. However, Gerry Weber's CIO actively invited Digitalcourage (2011) to assess their DBS (step 3). In our model, Digitalcourage is an NGO that represents civil society stakeholders potentially affected by this business decision, i.e. customers. In reconstructing the actual debate, we further draw on our model's ethical assessment triangle (step 4). For Gerry Weber's perspective (Figure 2, leftmost triangle), we get a high positive rating on the economic dimension due to the promise of substantial savings along the supply chain and additional revenue due to better product availability. On the ecological and social dimensions, we get slightly positive ratings, since optimized supply chain management implies less delivery vehicle emissions and also less employee stress due to a reduced need for manual stocktaking over weekends. From Digitalcourage's perspective, we get a slightly positive rating on the economic dimension (a positive economic effect for Gerry Weber may lead to lower prices for clothes) and a similarly slightly positive rating on the ecological dimension (reflecting the possible greener logistics) 
but a substantially negative rating on the social dimension, due to the negative effects on personal privacy (Figure 2, second triangle). Since we have a negative rating in one dimension for one stakeholder, a discourse is necessary in order to seek modifications to and, ultimately, a legitimization of the DBS.

During the discourse (step 5), Gerry Weber reportedly made several changes to its RFID implementation strategy (step 6), including not storing or using any customer-related information together with the RFID tag, openly informing its customers and educating the sales staff, offering to remove the tag during check-out and following strict privacy policies, including certifications and publishing the corresponding audits (Grone 2012). In our model, these changes would slightly lower the rating on the economic dimension (note that the cited presentation explicitly states that $99 \%$ of benefits are before check-out), whereas the other dimensions would remain unchanged (Figure 2, compare the first with the third triangle). For Digitalcourage, the substantially negative rating on the social dimension would have changed to a moderately negative rating (Figure 2, compare the second with the fourth triangle): While it positively acknowledged the other steps Gerry Weber took, any RFID chip in a sales area still constitutes a "no-go" for this NGO (Digitalcourage e.V. 2011). In the end, the discourse ended with a state that we characterize as agree-to-disagree, as Gerry Weber went ahead with implementing its modified RFID strategy (step 7c). Thus, we end the discourse with a stakeholder's assessment triangle where one of the areas still extends into the negative (although less so than before the start of the consensus process) and a non-legitimized DBS implementation decision.

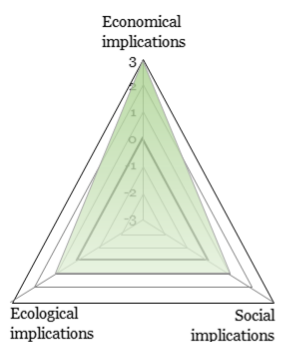

GERRY WEBER

Initial Assessment

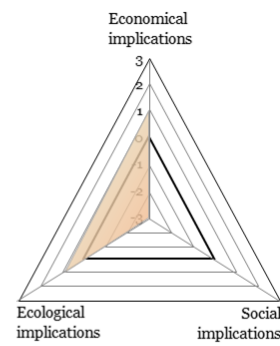

Digitalcourage Initial Assessment

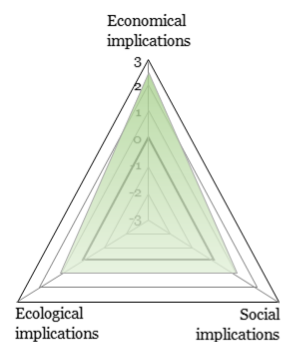

GERRY WEBER After Discourse

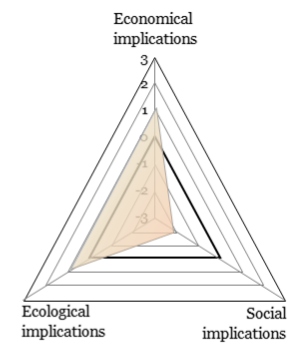

Digitalcourage After Discourse

\section{Figure 2: The reconstructed ethical assessment triangles for the Gerry Weber case}

In contrast to the Peuterey case, the discourse here has managed to lessen the perceived negative social implications (Figure 2, compare the second with the fourth triangle). Moreover, we are also able to point out explicit remaining questions for a further discourse among the different stakeholder groups. For the affected industries (fashion and logistics), the lack of consensus points to the need to consider revising the existing self-commitment policy for all RFID operators that existed back then (step 8c) (Grone 2012). For the government, a political question arises as to whether and how personal privacy needs in the context of RFID usage need to be regulated further (step 8b), since industry and society seem to have differing values that they are unable to reconcile on their own. For the affected society as a whole, the discourse objective is to assess whether and to what extent it is socially acceptable to digitally tag objects at all, given the danger that data and personal privacy may be compromised even if countermeasures are taken (step 8a). Lastly, from a more fundamental perspective, we have one planned action that one stakeholder assesses as ethical and another assesses as unethical - thus revealing the need for further philosophical debate (step 8d).

\section{Discussion and Conclusion}

With this paper, we contribute to the pragmatization of generic proposals from business and discourse ethics to applied IS ethics in the form of a model for ethical DBS development. Ethical considerations are of particular importance for DBS since their formulation is normally driven solely by economic interests, but their outcomes based on new technological developments may profoundly influence individuals, organizations, industries and societies. Our model draws on integrative business ethics (Ulrich 2008), which, in a nutshell, proposes the integration of an ethical discourse into organizational decision-making, so that the ultimate decision to pursue a particular DBS is made on the grounds of an achieved consensus among affected stakeholders.

Such a perspective integrating DBS and ethics extends and complements extant perspectives on ethical IS design and development. In particular, our approach precedes the IS design and development phase and simultaneously sets the stage for it during the subsequent DBS implementation. In other words, IT 
and business executives - who have the power and the mandate to support and block IT-related decisions with ethical implications - are involved at an early point in time before any IS development starts. In so doing, they can avoid having to deal with negative fallout when ethical issues arise during or after design, development or deployment. Thus, they can save resources on the design of information systems that are not feasible from an ethical standpoint and, in the worst case, may even have to be undeployed. In an ex-post application, as we did in section 4, one can also use the model to analyse past controversial IS-related business decisions and comprehensively highlight ethical aspects.

Since this is only a research-in-progress paper, there are several limitations. First, we selected the underlying business ethics literature pragmatically, settling on Ulrich's (2008) integrative business ethics as a convincing and well-argued foundation. Based on this pragmatic selection of literature, we developed an initial model striving to highlight the potential that even such an initial pragmatization can have. We evaluated the model only ex-post with examples of less disruptive DBS and have not yet demonstrated the actual utility for the ex-ante digital strategy development process. More generally, the model does not account for the disruptive nature of digital innovations where no one is able to reliably anticipate - and thus debate - these innovations' consequences and corresponding ethical implications.

Further research can incorporate other abstract advice from business ethics or other discourses into the model and thus make this advice more actionable. Further research can also validate the thus refined model with other, more complex cases. There is also a need to further develop and evaluate the model so that business and IT executives find it genuinely useful. This includes additional attention on ways and challenges to create the necessary institutional prerequisites such as appropriate management systems and a matching organizational culture. On a more theoretical level, further research can also investigate ways to resolve the inherent tensions between quick and wide-ranging DBS development on the one hand and the need to achieve consensus on ethical matters on the other hand, through either procedural or more fundamental means.

\section{References}

Apel, K.-O. 1980. Towards a Transformation of Philosophy, Marquette University Press.

Beuth, P. 2012. "Funketiketten: Datenschützer lesen RFID-Chips in Kleidung aus," Die Zeit, Hamburg (available at http://www.zeit.de/digital/datenschutz/2012-01/foebud-rfid-gerry-weber).

Bharadwaj, A., El Sawy, O. A., Pavlou, P. A., and Venkatraman, N. 2013. "Digital business strategy: Toward a next generation of insights," MIS Quarterly (37:2), pp. 471-482.

Digitalcourage e.V. 2011. "Technik: Peuterey | BigBrotherAwards," (available at http://bigbrotherawards.de/2011/technik-peuterey; retrieved July 13, 2016).

Grone, C. von. 2012.RFID@Gerry Weber.Fashion trifft Logistikwelt, (available at http://www.logistikheute.de/sites/default/files/logistik-

heute/fachforen/vortrag_rfid_in_der_textillogistik_optimierungsm_20614.pdf).

Grover, V., and Kohli, R. 2013. "REVEALING YOUR HAND: CAVEATS IN IMPLEMENTING DIGITAL BUSINESS STRATEGY.," Mis Quarterly (37:2).

Habermas, J. 1990. Moral Consciousness and Communicative Action, MIT Press.

Marrewijk, M. van. 2003. "Concepts and Definitions of CSR and Corporate Sustainability: Between Agency and Communion," Journal of Business Ethics (44:2-3), pp. 95-105.

Mingers, J., and Walsham, G. 2010. "Toward ethical information systems: the contribution of discourse ethics," Mis Quarterly (34:4), pp. 833-854.

Myers, M. D., and Venable, J. R. 2014. "A set of ethical principles for design science research in information systems," Information \& Management (51:6), pp. 801-809.

Ulrich, P. 2008. Integrative Economic Ethics: Foundations of a Civilized Market Economy, Cambridge, GB: Cambridge University Press.

\section{Copyright}

Copyright: (C) 2016 Oliver Degner \& Andreas Drechsler. This is an open-access article distributed under the terms of the Creative Commons Attribution-NonCommercial 3.0 Australia License, which permits non-commercial use, distribution, and reproduction in any medium, provided the original author and ACIS are credited. 